International Mathematical Forum, 1, 2006, no. 32, 1543 - 1554

\title{
A FIXED POINT THEOREM IN MENGER SPACES
}

\author{
Servet Kutukcu \\ Department of Mathematics, Faculty of Science and Arts, \\ Ondokuz Mayis University, Kurupelit, 55139 Samsun, Turkey
}

\begin{abstract}
In the present work, we prove a fixed point theorem in Menger spaces through weak compatibility.
\end{abstract}

Mathematics Subject Classification: 47H10, 54H25.

Keywords: Menger space, t-norm, Common fixed point, Compatible maps, Weak-compatible maps.

\section{Introduction}

There have been a number of generalizations of metric space. One such generalization is Menger space introduced in 1942 by Menger [10] who was use distribution functions instead of nonnegative real numbers as values of the metric. Schweizer and Sklar [16] studied this concept and then the important development of Menger space theory was due to Sehgal and Bharucha-Reid [13].

Sessa [14] introduced weakly commuting maps in metric spaces. Jungck [6] enlarged this concept to compatible maps. The notion of compatible maps in Menger spaces has been introduced by Mishra [11]. Recently, Singh and Jain [15] generalized the results of Mishra [11] using the concept of weak compatibility and compatibility of pair of self maps.

In this paper, using the idea of weak compatibility due to Singh and Jain [15] and the idea of compatibility due to Mishra [11], we prove a common fixed point theorem for six maps under the condition of weak compatibility and compatibility in Menger spaces and give an example to illustrate the theorem.

\section{Preliminary Notes}

In this section, we recall some definitions and known results in Menger space. For more details we refer the readers to $[1-5,7,8,9,12,17]$. 
Definition 2.1 A triangular norm * (shorty t-norm) is a binary operation on the unit interval $[0,1]$ such that for all $a, b, c, d \in[0,1]$ the following conditions are satisfied:

(a) $a * 1=a$;

(b) $a * b=b * a$;

(c) $a * b \leq c * d$ whenever $a \leq c$ and $b \leq d$;

(d) $a *(b * c)=(a * b) * c$.

Examples of t-norms are $a * b=\max \{a+b-1,0\}$ and $a * b=\min \{a, b\}$.

Definition 2.2 A distribution function is a function $F:[-\infty, \infty] \rightarrow[0,1]$ which is left continuous on $\mathbb{R}$, non-decreasing and $F(-\infty)=0, F(\infty)=1$.

We will denote by $\Delta$ the family of all distribution functions on $[-\infty, \infty]$. $H$ is a special element of $\Delta$ defined by

$$
H(t)=\left\{\begin{array}{ll}
0 & \text { if } t \leq 0, \\
1 & \text { if } t>0 .
\end{array} .\right.
$$

If $X$ is a nonempty set, $F: X \times X \rightarrow \Delta$ is called a probabilistic distance on $X$ and $F(x, y)$ is usually denoted by $F_{x y}$.

Definition 2.3 (Schweizer and Sklar [16]) The ordered pair $(X, F)$ is called a probabilistic metric space (shortly PM-space) if $X$ is a nonempty set and $F$ is a probabilistic distance satisfying the following conditions: for all $x, y, z \in X$ and $t, s>0$,

$(\mathrm{FM}-0) F_{x y}(t)=1 \Longleftrightarrow x=y$

$(\mathrm{FM}-1) F_{x y}(0)=0$;

(FM-2) $F_{x y}=F_{y x}$;

$(\mathrm{FM}-3) F_{x z}(t)=1, F_{z y}(s)=1 \Rightarrow F_{x y}(t+s)=1$.

The ordered triple $(X, F, *)$ is called Menger space if $(X, F)$ is a PM-space, $*$ is a t-norm and the following condition is also satisfies: for all $x, y, z \in X$ and $t, s>0$,

$(\mathrm{FM}-4) F_{x y}(t+s) \geq F_{x z}(t) * F_{z y}(s)$.

Proposition 2.4 (Sehgal and Bharucha-Reid [13]) Let $(X, d)$ be a metric space. Then the metric $d$ induces a distribution function $F$ defined by $F_{x y}(t)=H(t-d(x, y))$ for all $x, y \in X$ and $t>0$. If t-norm $*$ is $a * b=$ $\min \{a, b\}$ for all $a, b \in[0,1]$ then $(X, F, *)$ is a Menger space. Further, $(X, F, *)$ is a complete Menger space if $(X, d)$ is complete.

Definition 2.5 (Mishra [11]) Let $(X, F, *)$ be a Menger space and $*$ be a continuous t-norm. 
(a) A sequence $\left\{x_{n}\right\}$ in $X$ is said to be converge to a point $x$ in $X$ (written $\left.x_{n} \rightarrow x\right)$ iff for every $\epsilon>0$ and $\lambda \in(0,1)$, there exists an integer $n_{0}=n_{0}(\varepsilon, \lambda)$ such that $F_{x_{n} x}(\varepsilon)>1-\lambda$ for all $n \geq n_{0}$.

(b) A sequence $\left\{x_{n}\right\}$ in $X$ is said to be Cauchy if for every $\epsilon>0$ and $\lambda \in(0,1)$, there exists an integer $n_{0}=n_{0}(\varepsilon, \lambda)$ such that $F_{x_{n} x_{n+p}}(\varepsilon)>1-\lambda$ for all $n \geq n_{0}$ and $p>0$.

(c) A Menger space in which every Cauchy sequence is convergent is said to be complete.

Remark 2.6 If $*$ is a continuous t-norm, it follows from (FM-4) that the limit of sequence in Menger space is uniquely determined.

Definition 2.7 (Singh and Jain [15]) Self maps $A$ and $B$ of a Menger space $(X, F, *)$ are said to be weakly compatible (or coincidentally commuting) if they commute at their coincidence points, i.e. if $A x=B x$ for some $x \in X$ then $A B x=B A x$.

Definition 2.8 (Mishra [11]) Self maps $A$ and $B$ of a Menger space $(X$, $F, *)$ are said to be compatible if $F_{A B x_{n} B A x_{n}}(t) \rightarrow 1$ for all $t>0$, whenever $\left\{x_{n}\right\}$ is a sequence in $X$ such that $A x_{n}, B x_{n} \rightarrow x$ for some $x$ in $X$ as $n \rightarrow \infty$.

Remark 2.9 If self maps $A$ and $B$ of a Menger space $(X, F, *)$ are compatible then they are weakly compatible.

The following is an example of pair of self maps in a Menger space which are weakly compatible but not compatible.

Example 2.10 Let $(X, d)$ be a metric space where $X=[0,2]$ and $(X, F, *)$ be the induced Menger space with $F_{x y}(t)=H(t-d(x, y)), \forall x, y \in X$ and $\forall t>0$. Define self maps $A$ and $B$ as follows:

$$
A x=\left\{\begin{array}{ll}
2-x, & \text { if } 0 \leq x<1, \\
2, & \text { if } 1 \leq x \leq 2,
\end{array} \quad \text { and } B x= \begin{cases}x, & \text { if } 0 \leq x<1, \\
2, & \text { if } 1 \leq x \leq 2 .\end{cases}\right.
$$

Take $x_{n}=1-1 / n$. Then $F_{A x_{n} 1}(t)=H(t-(1 / n))$ and $\lim _{n \rightarrow \infty} F_{A x_{n} 1}(t)=H(t)=$ 1. Hence $A x_{n} \rightarrow \infty$ as $n \rightarrow \infty$. Similarly, $B x_{n} \rightarrow \infty$ as $n \rightarrow \infty$. Also $F_{A B x_{n} B A x_{n}}(t)=H(t-(1-1 / n))$ and $\lim _{n \rightarrow \infty} F_{A B x_{n} B A x_{n}}(t)=H(t-1) \neq 1$, $\forall t>0$. Hence the pair $(A, B)$ is not compatible. Set of coincidence points of $A$ and $B$ is $[1,2]$. Now for any $x \in[1,2], A x=B x=2$, and $A B(x)=A(2)=$ $2=S(2)=S A(x)$. Thus $A$ and $B$ are weakly compatible but not compatible.

Lemma 2.11 (Singh and Jain [15]) Let $\left\{x_{n}\right\}$ be a sequence in a Menger space $(X, F, *)$ with continuous $t$-norm $*$ and $t * t \geq t$. If there exists a constant $k \in(0,1)$ such that

$$
F_{x_{n} x_{n+1}}(k t) \geq F_{x_{n-1} x_{n}}(t)
$$

for all $t>0$ and $n=1,2 \ldots$, then $\left\{x_{n}\right\}$ is a Cauchy sequence in $X$. 
Lemma 2.12 (Singh and Jain [15]) Let $(X, F, *)$ be a Menger space. If there exists $k \in(0,1)$ such that

$$
F_{x y}(k t) \geq F_{x y}(t)
$$

for all $x, y \in X$ and $t>0$, then $x=y$.

\section{Main Results}

Theorem 3.1 Let $A, B, S, T, L$ and $M$ be self maps on a complete Menger space $(X, F, *)$ with $t * t \geq t$ for all $t \in[0,1]$, satisfying:

(a) $L(X) \subseteq S T(X), M(X) \subseteq A B(X)$;

(b) there exists a constant $k \in(0,1)$ such that

$$
\begin{aligned}
& F_{L x M y}^{2}(k t) *\left[F_{A B x L x}(k t) \cdot F_{S T y M y}(k t)\right] \\
\geq & {\left[p F_{A B x L x}(t)+q F_{A B x S T y}(t)\right] \cdot F_{A B x M y}(2 k t) }
\end{aligned}
$$

for all $x, y \in X$ and $t>0$ where $0<p, q<1$ such that $p+q=1$;

(c) $A B=B A, S T=T S, L B=B L, M T=T M$;

(d) either $A B$ or $L$ is continuous;

(e) the pair $(L, A B)$ is compatible and $(M, S T)$ is weakly compatible.

Then $A, B, S, T, L$ and $M$ have a unique common fixed point.

Proof. Let $x_{0}$ be an arbitrary point of $X$. By (a), there exists $x_{1}, x_{2} \in X$ such that $L x_{0}=S T x_{1}=y_{0}$ and $M x_{1}=A B x_{1}=y_{1}$. Inductively, we can construct sequences $\left\{x_{n}\right\}$ and $\left\{y_{n}\right\}$ in $X$ such that $L x_{2 n}=S T x_{2 n+1}=y_{2 n}$ and $M x_{2 n+1}=A B x_{2 n+2}=y_{2 n+1}$ for $n=0,1,2, \ldots$.

Step1. By taking $x=x_{2 n}$ and $y=x_{2 n+1}$ in (b), we have

$$
\begin{gathered}
F_{L x_{2 n} M x_{2 n+1}}^{2}(k t) *\left[F_{A B x_{2 n} L x_{2 n}}(k t) F_{S T x_{2 n+1} M x_{2 n+1}}(k t)\right] \\
\geq\left[p F_{A B x_{2 n} L x_{2 n}}(t)+q F_{A B x_{2 n} S T x_{2 n+1}}(t)\right] F_{A B x_{2 n} M x_{2 n+1}}(2 k t), \\
F_{y_{2 n} y_{2 n+1}}^{2}(k t) *\left[F_{y_{2 n-1} y_{2 n}}(k t) F_{y_{2 n} y_{2 n+1}}(k t)\right] \\
\geq\left[p F_{y_{2 n} y_{2 n-1}}(t)+q F_{y_{2 n-1} y_{2 n}}(t)\right] F_{y_{2 n-1} y_{2 n+1}}(2 k t), \\
F_{y_{2 n} y_{2 n+1}}(k t)\left[F_{y_{2 n-1} y_{2 n}}(k t) * F_{y_{2 n} y_{2 n+1}}(k t)\right] \\
\geq(p+q) F_{y_{2 n} y_{2 n-1}}(t) F_{y_{2 n-1} y_{2 n+1}}(2 k t),
\end{gathered}
$$




$$
F_{y_{2 n} y_{2 n+1}}(k t) F_{y_{2 n-1} y_{2 n+1}}(2 k t) \geq F_{y_{2 n-1} y_{2 n}}(t) F_{y_{2 n-1} y_{2 n+1}}(2 k t) .
$$

Hence, we have

$$
F_{y_{2 n} y_{2 n+1}}(k t) \geq F_{y_{2 n-1} y_{2 n}}(t) .
$$

Similarly, we also have

$$
F_{y_{2 n+1} y_{2 n+2}}(k t) \geq F_{y_{2 n} y_{2 n+1}}(t) .
$$

In general, for all $n$ even or odd, we have

$$
F_{y_{n} y_{n+1}}(k t) \geq F_{y_{n-1} y_{n}}(t)
$$

for $k \in(0,1)$ and all $t>0$. Thus, by Lemma 2.11, $\left\{y_{n}\right\}$ is a Cauchy sequence in $X$. Since $(X, F, *)$ is complete, it converges to a point $z$ in $X$. Also its subsequences converge as follows: $\left\{L x_{2 n}\right\} \rightarrow z,\left\{A B x_{2 n}\right\} \rightarrow z$, $\left\{M x_{2 n+1}\right\} \rightarrow z$ and $\left\{S T x_{2 n+1}\right\} \rightarrow z$.

Case I. $A B$ is continuous. Since $A B$ is continuous, $A B(A B) x_{2 n} \rightarrow A B z$ and $(A B) L x_{2 n} \rightarrow A B z$. Since $(L, A B)$ is compatible, $L(A B) x_{2 n} \rightarrow A B z$.

Step 2. By taking $x=A B x_{2 n}$ and $y=x_{2 n+1}$ in (b), we have

$$
\begin{aligned}
& F_{L(A B) x_{2 n} M x_{2 n+1}}^{2}(k t) *\left[F_{A B(A B) x_{2 n} L(A B) x_{2 n}}(k t) F_{S T x_{2 n+1} M x_{2 n+1}}(k t)\right] \\
\geq & {\left[p F_{A B(A B) x_{2 n} L(A B) x_{2 n}}(t)+q F_{A B(A B) x_{2 n} S T x_{2 n+1}}(t)\right] F_{A B(A B) x_{2 n} M x_{2 n+1}} }
\end{aligned}
$$

This implies that, as $n \rightarrow \infty$

$$
\begin{aligned}
F_{z A B z}^{2}(k t) *\left[F_{A B z A B z}(k t) F_{z z}(k t)\right] & \geq\left[p F_{A B z A B z}(t)+q F_{z A B z}(t)\right] F_{z A B z}(2 k t) \\
& \geq\left[p+q F_{z A B z}(t)\right] F_{z A B z}(k t), \\
F_{z A B z}(k t) & \geq p+q F_{z A B z}(t) \\
\geq & p+q F_{z A B z}(k t), \\
F_{z A B z}(k t) & \geq \frac{p}{1-q}=1
\end{aligned}
$$

for $k \in(0,1)$ and all $t>0$. Thus, we have $z=A B z$.

Step 3. By taking $x=z$ and $y=x_{2 n+1}$ in (b), we have

$$
\begin{aligned}
& F_{L z M x_{2 n+1}}^{2}(k t) *\left[F_{A B z L z}(k t) F_{S T x_{2 n+1} M x_{2 n+1}}(k t)\right] \\
\geq & {\left[p F_{A B z L z}(t)+q F_{A B z S T x_{2 n+1}}(t)\right] F_{A B z M x_{2 n+1}}(2 k t) . }
\end{aligned}
$$


This implies that, as $n \rightarrow \infty$

$$
\begin{aligned}
F_{z L z}^{2}(k t) *\left[F_{z L z}(k t) F_{z z}(k t)\right] & \geq\left[p F_{z L z}(t)+q F_{z z}(t)\right] F_{z z}(2 k t) \\
F_{z L z}^{2}(k t) * F_{z L z}(k t) & \geq p F_{z L z}(t)+q
\end{aligned}
$$

Noting that $F_{z L z}^{2}(k t) \leq 1$ and using (c) in Definition 2.1, we have

$$
\begin{aligned}
F_{z L z}(k t) & \geq p F_{z L z}(t)+q \\
& \geq p F_{z L z}(k t)+q \\
F_{z L z}(k t) & \geq \frac{q}{1-p}=1
\end{aligned}
$$

for $k \in(0,1)$ and all $t>0$. Thus, we have $z=L z=A B z$.

Step 4. By taking $x=B z, y=x_{2 n+1}$ with $\alpha=1$ in (b), we have

$$
\begin{aligned}
& F_{L(B z) M x_{2 n+1}}^{2}(k t) *\left[F_{A B(B z) L(B z)}(k t) F_{S T x_{2 n+1} M x_{2 n+1}}(k t)\right] \\
& \geq \quad\left[p F_{A B(B z) L(B z)}(t)+q F_{A B(B z) S T x_{2 n+1}}(t)\right] F_{A B(B z) M x_{2 n+1}}(2 k t) .
\end{aligned}
$$

Since $A B=B A$ and $B L=L B$, we have $L(B z)=B(L z)=B z$ and $A B(B z)=B(A B z)=B z$. Letting $n \rightarrow \infty$, we have

$$
\begin{aligned}
F_{z B z}^{2}(k t) *\left[F_{B z B z}(k t) F_{z z}(k t)\right] & \geq\left[p F_{B z B z}(t)+q F_{z B z}(t)\right. \\
F_{z B z}^{2}(k t) \geq\left[p+q F_{z B z}(t)\right] F_{z B z}(2 k t) & \geq\left[p+q F_{z B z}(t)\right] F_{z B z}(k t), \\
& \\
F_{z B z}(k t) & \geq p+q F_{z B z}(t) \\
& \geq p+q F_{z B z}(k t), \\
F_{z B z}(k t) & \geq \frac{p}{1-q}=1
\end{aligned}
$$

for $k \in(0,1)$ and all $t>0$. Thus, we have $z=B z$. Since $z=A B z$, we also have $z=A z$. Therefore, $z=A z=B z=L z$.

Step 5. Since $L(X) \subseteq S T(X)$, there exists $v \in X$ such that $z=L z=$ $S T v$. By taking $x=x_{2 n}, y=v$ in (b), we have

$$
\begin{aligned}
& F_{L x_{2 n} M v}^{2}(k t) *\left[F_{A B x_{2 n} L x_{2 n}}(k t) F_{S T v M v}(k t)\right] \\
\geq & {\left[p F_{A B x_{2 n} L x_{2 n}}(t)+q F_{A B x_{2 n} S T v}(t)\right] F_{A B x_{2 n} M v}(2 k t) }
\end{aligned}
$$


which implies that, as $n \rightarrow \infty$

$$
\begin{gathered}
F_{z M v}^{2}(k t) *\left[F_{z z}(k t) F_{z M v}(k t)\right] \geq\left[p F_{z z}(t)+q F_{z z}(t)\right] F_{z M v}(2 k t), \\
F_{z M v}^{2}(k t) * F_{z M v}(k t) \geq(p+q) F_{z M v}(2 k t) .
\end{gathered}
$$

Noting that $F_{z M v}^{2}(k t) \leq 1$ and using (c) in Definition 2.1, we have

$$
\begin{aligned}
F_{z M v}(k t) & \geq F_{z M v}(2 k t) \\
& \geq F_{z M v}(t)
\end{aligned}
$$

Thus, by Lemma 2.12, we have $z=M v$ and so $z=M v=S T v$. Since $(M, S T)$ is weakly compatible, we have $S T M v=M S T v$. Thus, $S T z=$ $M z$.

Step 6. By taking $x=x_{2 n}, y=z$ in (b) and using Step 5, we have

$$
\begin{aligned}
& F_{L x_{2 n} M z}^{2}(k t) *\left[F_{A B x_{2 n} L x_{2 n}}(k t) F_{S T z M z}(k t)\right] \\
\geq & {\left[p F_{A B x_{2 n} L x_{2 n}}(t)+q F_{A B x_{2 n} S T z}(t)\right] F_{A B x_{2 n} M z}(2 k t) }
\end{aligned}
$$

which implies that, as $n \rightarrow \infty$

$$
\begin{aligned}
& F_{z M z}^{2}(k t) *\left[F_{z z}(k t) F_{M z M z}(k t)\right] \geq\left[p F_{z z}(t)+q F_{z M z}(t)\right] F_{z M z}(2 k t), \\
& F_{z M z}^{2}(k t) \geq\left[p+q F_{z M z}(t)\right] F_{z M z}(2 k t) \\
& \geq\left[p+q F_{z M z}(t)\right] F_{z M z}(k t) \\
& F_{z M z}(k t) \\
& \geq p+q F_{z M z}(t) \\
& \geq p+q F_{z M z}(k t) \\
& F_{z M z}(k t) \geq \frac{p}{1-q}=1 .
\end{aligned}
$$

Thus, we have $z=M z$ and therefore $z=A z=B z=L z=M z=S T z$.

Step 7. By taking $x=x_{2 n}, y=T z$ in (b), we have

$$
\begin{aligned}
& F_{L x_{2 n} M(T z)}^{2}(k t) *\left[F_{A B x_{2 n} L x_{2 n}}(k t) F_{S T(T z) M(T z)}(k t)\right] \\
& \geq \quad\left[p F_{A B x_{2 n} L x_{2 n}}(t)+q F_{A B x_{2 n} S T(T z)}(t)\right] F_{A B x_{2 n} M(T z)}(2 k t) .
\end{aligned}
$$

Since $M T=T M$ and $S T=T S$, we have $M T z=T M z=T z$ and $S T(T z)=T(S T z)=T z$. Letting $n \rightarrow \infty$, we have

$$
F_{z T z}^{2}(k t) *\left[F_{z z}(k t) F_{T z T z}(k t)\right] \geq\left[p F_{z z}(t)+q F_{z T z}(t)\right] F_{z T z}(2 k t),
$$




$$
\begin{aligned}
F_{z T z}^{2}(k t) \geq\left[p+q F_{z T z}(t)\right] F_{z T z}(k t), & \\
F_{z T z}(k t) & \geq p+q F_{z T z}(t) \\
& \geq p+q F_{z T z}(k t), \\
F_{z T z}(k t) & \geq \frac{p}{1-q}=1 .
\end{aligned}
$$

Thus, we have $z=T z$. Since $T z=S T z$, we also have $z=S z$. Therefore, $z=A z=B z=L z=M z=S z=T z$, that is, $z$ is the common fixed point of the six maps.

Case II. $L$ is continuous. Since $L$ is continuous, $L L x_{2 n} \rightarrow L z$ and $L(A B) x_{2 n} \rightarrow L z$. Since $(L, A B)$ is compatible, $(A B) L x_{2 n} \rightarrow L z$.

Step 8. By taking $x=L x_{2 n}, y=x_{2 n+1}$ in (b), we have

$$
\begin{aligned}
& F_{L L x_{2 n} M x_{2 n+1}}^{2}(k t) *\left[F_{A B L x_{2 n} L L x_{2 n}}(k t) F_{S T x_{2 n+1} M x_{2 n+1}}(k t)\right] \\
\geq & {\left[p F_{A B L x_{2 n} L L x_{2 n}}(t)+q F_{A B L x_{2 n} S T x_{2 n+1}}(t)\right] F_{A B L x_{2 n} M x_{2 n+1}}(2 k t) . }
\end{aligned}
$$

This implies that, as $n \rightarrow \infty$

$$
\begin{aligned}
& F_{z L z}^{2}(k t) *\left[F_{L z L z}(k t) F_{z z}(k t)\right] \geq\left[p F_{L z L z}(t)+q F_{z L z}(t)\right] F_{z L z}(2 k t), \\
& F_{z L z}^{2}(k t) \geq\left[p+q F_{z L z}(t)\right] F_{z L z}(2 k t) \\
& \geq\left[p+q F_{z L z}(t)\right] F_{z L z}(k t) \\
& F_{z L z}(k t) \geq p+q F_{z L z}(t) \\
& \geq p+q F_{z L z}(k t) \\
& F_{z L z}(k t) \geq \frac{p}{1-q}=1 .
\end{aligned}
$$

Thus, we have $z=L z$ and using Steps 5 -7, we have $z=L z=M z=$ $S z=T z$.

Step 9. Since $M(X) \subseteq A B(X)$, there exists $v \in X$ such that $z=M z=$ $A B v$. By taking $x=v, y=x_{2 n+1}$ in (b), we have

$$
\begin{aligned}
& F_{L v M x_{2 n+1}}^{2}(k t) *\left[F_{A B v L v}(k t) F_{S T x_{2 n+1} M x_{2 n+1}}(k t)\right] \\
\geq & {\left[p F_{A B v L v}(t)+q F_{A B v S T x_{2 n+1}}(t)\right] F_{A B v M x_{2 n+1}}(2 k t) }
\end{aligned}
$$


which implies that, as $n \rightarrow \infty$

$$
\begin{aligned}
F_{z L v}^{2}(k t) *\left[F_{z L v}(k t) F_{z z}(k t)\right] \geq & {\left[p F_{z L v}(t)+q F_{z z}(t)\right] F_{z z}(2 k t), } \\
F_{z L v}^{2}(k t) * F_{z L v}(k t) & \geq p F_{z L v}(t)+q \\
& \geq p F_{z L v}(k t)+q .
\end{aligned}
$$

Noting that $F_{z L v}^{2}(k t) \leq 1$ and using $(\mathrm{c})$ in Definition 2.1, we have

$$
\begin{gathered}
F_{z M v}(k t) \geq p F_{z L v}(k t)+q, \\
F_{z M v}(k t) \geq \frac{q}{1-p}=1 .
\end{gathered}
$$

Thus, we have $z=L v=A B v$. Since $(L, A B)$ is weakly compatible, we have $L z=A B z$ and using Step 4 , we also have $z=B z$. Therefore $z=A z=B z=S z=T z=L z=M z$, that is, $z$ is the common fixed point of the six maps in this case also.

Step 10. For uniqueness, let $w(w \neq z)$ be another common fixed point of $A, B, S, T, L$ and $M$. Taking $x=z, y=w$ in (b), we have

$$
\begin{aligned}
& F_{L z M w}^{2}(k t) *\left[F_{A B z L z}(k t) F_{S T w M w}(k t)\right] \\
\geq & {\left[p F_{A B z L z}(t)+q F_{A B z S T w}(t)\right] F_{A B z M w}(2 k t) }
\end{aligned}
$$

which implies that

$$
\begin{aligned}
F_{z w}^{2}(k t) & \geq\left[p+q F_{z w}(t)\right] F_{z w}(2 k t) \\
\geq & {\left[p+q F_{z w}(t)\right] F_{z w}(k t), } \\
F_{z w}(k t) & \geq p+q F_{z w}(t) \\
& \geq p+q F_{z w}(k t), \\
F_{z w}(k t) & \geq \frac{p}{1-q}=1 .
\end{aligned}
$$

Thus, we have $z=w$. This completes the proof of the theorem.

If we take $B=T=I_{X}$ ( the identity map on $X$ ) in the main Theorem, we have the following:

Corollary 3.2 Let $A, S, L$ and $M$ be self maps on a complete Menger space $(X, F, *)$ with $t * t \geq t$ for all $t \in[0,1]$, satisfying: 
(a) $L(X) \subseteq S(X), M(X) \subseteq A(X)$;

(b) there exists a constant $k \in(0,1)$ such that

$$
\begin{aligned}
& F_{L x M y}^{2}(k t) *\left[F_{A x L x}(k t) \cdot F_{S y M y}(k t)\right] \\
\geq & {\left[p F_{A x L x}(t)+q F_{A x S y}(t)\right] \cdot F_{A x M y}(2 k t) }
\end{aligned}
$$

for all $x, y \in X$ and $t>0$ where $0<p, q<1$ such that $p+q=1$;

(c) either $A$ or $L$ is continuous;

(d) the pair $(L, A)$ is compatible and $(M, S)$ is weakly compatible.

Then $A, S, L$ and $M$ have a unique common fixed point.

If we take $A=S, L=M$ and $B=T=I_{X}$ in the main Theorem, we have the following:

Corollary 3.3 Let $(X, F, *)$ be a complete Menger space with $t * t \geq t$ for all $t \in[0,1]$ and let $A$ and $L$ be compatible maps on $X$ such that $L(X) \subseteq A(X)$. If $A$ is continuous and there exists a constant $k \in(0,1)$ such that

$$
\begin{aligned}
& F_{L x L y}^{2}(k t) *\left[F_{A x L x}(k t) \cdot F_{A y L y}(k t)\right] \\
\geq & {\left[p F_{A x L x}(t)+q F_{A x A y}(t)\right] \cdot F_{A x L y}(2 k t) }
\end{aligned}
$$

for all $x, y \in X$ and $t>0$ where $0<p, q<1$ such that $p+q=1$, then $A$ and $L$ have a unique fixed point.

Example 3.4 Let $X=[0,1]$ with the metric d defined by $d(x, y)=|x-y|$ and define $F_{x y}(t)=H(t-d(x, y))$ for all $x, y \in X, t>0$. Clearly $(X, F, *)$ is a complete Menger space where $t$-norm $*$ is defined by $a * b=\min \{a, b\}$ for all $a, b \in[0,1]$. Let $A, B, S, T, L$ and $M$ be maps from $X$ into itself defined as

$$
A x=x, B x=\frac{x}{2}, S x=\frac{x}{5}, T x=\frac{x}{3}, L x=0, M x=\frac{x}{6}
$$

for all $x \in X$. Then

$$
L(X)=\{0\} \subset\left[0, \frac{1}{15}\right]=S T(X) \text { and } M(X)=\left[0, \frac{1}{6}\right] \subset\left[0, \frac{1}{2}\right]=A B(X) .
$$

Clearly $A B=B A, S T=T S, L B=B L, M T=T M$ and $A B, L$ are continuous. If we take $k=1 / 2$ and $t=1$, we see that the condition (b) of the main Theorem is also satisfied. Moreover, the maps $L$ and $A B$ are compatible if $\lim _{n \rightarrow \infty} x_{n}=0$, where $\left\{x_{n}\right\}$ is a sequence in $X$ such that $\lim _{n \rightarrow \infty} L x_{n}=$ $\lim _{n \rightarrow \infty} A B x_{n}=0$ for $0 \in X$. The maps $M$ and $S T$ are weakly compatible at 0 . Thus, all conditions of the main Theorem are satisfied and 0 is the unique common fixed point of $A, B, S, T, L$ and $M$. 


\section{References}

[1] G. Constantin, I. Istratescu, Elements of Probabilistic Analysis, Ed. Acad. Bucureşti and Kluwer Acad. Publ., 1989.

[2] O. Hadzic, Common fixed point theorems for families of mapping in complete metric space, Math. Japon., 29 (1984), 127-134.

[3] T. L. Hicks, Fixed point theory in probabilistic metric spaces, Rev. Res. Novi Sad, 13 (1983), 63-72.

[4] I. Istratescu, On some fixed point theorems in generalized Menger spaces, Boll. Un. Mat. Ital., 5 (13-A) (1976), 95-100.

[5] I. Istratescu, On generalized complete probabilistic metric spaces, Rev. Roum. Math. Pures Appl., XXV (1980), 1243-1247.

[6] G. Jungck, Commuting mappings and fixed points, Amer. Math. Monthly, 83 (1976), 261-263.

[7] G. Jungck, Compatible mappings and common fixed points, Internat. J. Math. Sci., 9 (1986), 771-779.

[8] G. Jungck, B. E. Rhoades, Some fixed point theorems for compatible maps, Internat. J. Math. Ef Math. Sci., 3 (1993), 417-428.

[9] S. Kutukcu, D. Turkoglu, C. Yildiz, Common fixed points of compatible maps of type $(\beta)$ on fuzzy metric spaces, Commun. Korean Math. Soc., in press.

[10] K. Menger, Statistical metric, Proc. Nat. Acad., 28 (1942), 535-537.

[11] S. N. Mishra, Common fixed points of compatible mappings in PMspaces,ic spaces, Math. Japon., 36 (1991), 283-289.

[12] E. Pap, O. Hadzic, R. Mesiar, A fixed point theorem in probabilistic metric spaces and an application, J. Math. Anal. Appl., 202 (1996), 433449 .

[13] V. M. Sehgal, A. T. Bharucha-Reid, Fixed point of contraction mapping on PM spaces, Math. Systems Theory, 6 (1972), 97-100.

[14] S. Sessa, On a weak commutative condition in fixed point consideration, Publ. Inst. Math., 32 (1982), 146-153.

[15] B. Singh, S. Jain, A fixed point theorem in Menger Space through weak compatibility, J. Math. Anal. Appl., 301 (2005), 439-448. 
[16] B. Schweizer, A. Sklar, Probabilistic Metric Spaces, North-Holland, Amsterdam, 1983.

[17] R. M. Tardiff, Contraction maps on probabilistic metric spaces, J. Math. Anal. Appl., 165 (1992), 517-523.

Received: December 9, 2005 